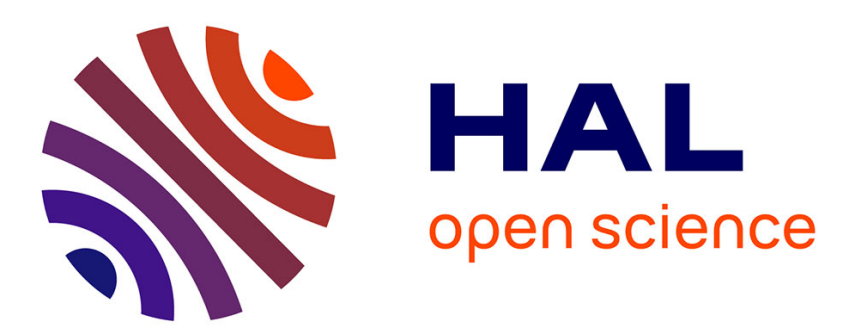

\title{
Scaling analysis of convective solute transport and segregation in Bridgman crystal growth from the doped melt \\ D. Camel, J.J. Favier
}

\section{- To cite this version:}

D. Camel, J.J. Favier. Scaling analysis of convective solute transport and segregation in Bridgman crystal growth from the doped melt. Journal de Physique, 1986, 47 (6), pp.1001-1014. 10.1051/jphys:019860047060100100 . jpa-00210276

\section{HAL Id: jpa-00210276 https://hal.science/jpa-00210276}

Submitted on 1 Jan 1986

HAL is a multi-disciplinary open access archive for the deposit and dissemination of scientific research documents, whether they are published or not. The documents may come from teaching and research institutions in France or abroad, or from public or private research centers.
L'archive ouverte pluridisciplinaire HAL, est destinée au dépôt et à la diffusion de documents scientifiques de niveau recherche, publiés ou non, émanant des établissements d'enseignement et de recherche français ou étrangers, des laboratoires publics ou privés. 


\title{
Scaling analysis of convective solute transport and segregation in Bridgman crystal growth from the doped melt
}

\author{
D. Camel and J. J. Favier \\ Laboratoire d'Etude de la Solidification, Département de Métallurgie, \\ Centre d'Etudes Nucléaires de Grenoble, 85 X, F-38041 Grenoble Cedex, France
}

(Reçu le 23 septembre 1985, révisé le 14 février 1986, accepté le 17 février 1986)

\begin{abstract}
Résumé. - Il est bien connu que le transport convectif a une influence décisive sur la macroségrégation observée dans les cristaux métalliques ou semi-conducteurs solidifiés à partir du bain fondu impur ou dopé. Nous étudions ce problème, dans le cas de la solidification Bridgman, à l'aide d'une analyse en ordres de grandeur des équations de bilan pour la masse, la quantité de mouvement et le soluté, dans le bain liquide et à l'interface. Nous définissons les différents régimes de transport de soluté et nous obtenons la loi d'échelle vérifiée dans chaque régime par l'étendue de la couche limite de soluté. Enfin, nous en déduisoms les différents types de macroségrégation dans le cristal. Nos prédictions sont en accord avec les résultats des simulations numériques et des expériences effectuées au sol ou en conditions de microgravité. Ainsi notre analyse fournit une méthode simple pour prédire la macroségrégation en fonction des propriétés physiques du système et des conditions expérimentales imposées : dimensions de l'échantillon, vitesse de croissance, gradients thermiques, orientation et niveau de gravité. Nous suggérons l'extension de notre méthode à d'autres configurations de croissance cristalline.
\end{abstract}

\begin{abstract}
Convective transport is well known to have a decisive influence on the macrosegregation encountered in metallic or semi-conductor crystals grown from an impure or doped melt. In the present paper, this problem is studied with the help of an order of magnitude analysis of the set of balance equations for mass, momentum and solute in the melt and at the growth interface. The different solute transport regimes are defined and the scaling laws of the solute boundary layer extent are obtained in each regime. Finally, the corresponding types of macrosegregation in the solidified crystal are derived. Our predictions compare favorably with the results of numerical simulations and ground-based as well as microgravity experiments. Thus our analysis provides a simple way to predict macrosegregation as a function of the physical properties of the system as well as the imposed experimental conditions : sample dimensions, growth rate, thermal gradients, gravity orientation and level. The extension of the method to other crystal growth situations is suggested.
\end{abstract}

\section{Introduction.}

The segregation of a dopant (or solute) in a crystal grown from its doped melt (or from a binary alloy) is well known to be mainly controlled by diffusion and/or convection in the melt (see for instance [1]). However, while diffusion laws can simply be accounted for, a very wide variety of convective phenomena may be encountered, according to the crystal growth method, boundary conditions, and physical system considered.

In their classical work [2], Burton, Prim and Slichter (BPS) worked out an analytical solution of this problem for the case of an idealized Czochralski growth configuration (infinite planar disk rotating above a semi-infinite melt). Following their work, the intuitive concept of a solute boundary layer was extended to other crystal growth configurations. According to this concept there is a limited region of extent $\delta$ in the liquid in front of the growth interface where solute transport occurs by diffusion; outside this region, the liquid is supposed to be homogenized by convection. Thus different situations are encountered according to the relative value of the characteristic length $\delta$ as compared with 1) the geometrical length of the liquid zone, and 2) the diffusion length of the considered species. Boundary layer models have been successfully generalized to predict segregation phenomena in various crystal growth configurations $[3,4]$. No general rule, however, has been given to derive $\delta$ from the data and boundary conditions of the problem. 
With the recent development of powerful numerical methods, a full treatment of complex cases has become possible. A number of results have already been obtained by this way [13-18]. However, as more and more complex problems are considered, some specific limitations of a purely numerical analysis appear. For instance : the step by step procedure which is inherent to numerical techniques limits the data range which can be scanned; simplifying physical assumptions can only be verified a posteriori; numerical difficulties appear in the case of rapid motions and/or boundary layer regimes.

Thus the object of the present paper is to show how scaling analysis can be a useful complement to the previous methods. Such an analysis can be based on the concepts of the boundary layer theory which has been largely developed for the case of external flows (flows around a solid body) [5]. Application of these concepts to internal flows (flows in enclosures) [6-7] has been more limited. We presently apply the same ideas to determine the occurrence and extent of solute boundary layers in crystal growth situations. This in fact proves very useful in crystal growth from the melt or solution where such boundary layers are very frequent due to the low chemical diffusivities.

The analysis proposed in the present paper generalizes our previous work [8] by considering the independent effect of the Grashof and Schmidt numbers and by giving consistent criteria to determine the practical characteristic quantities in the whole range of values of the parameters. We shall focus on the crystal growth of a doped metallic or semi-conductor crystal by the Bridgman method. In this simple case the flow decouples from the solutal field and is mainly driven by thermal buoyancy. The general physical assumptions and the equations governing momentum and solute transport will be recalled first. A scaling analysis will be performed and checked against the available numerical data in order to establish simple laws giving the main characteristics of the flow especially in the vicinity of the growth interface. The same scaling analysis method will then be applied to the solute field, leading to a general definition of the solute boundary layer in the melt in front of the growth interface, and a description of the different types of segregation obtained in the crystal. The predictions thus made will be compared with the numerical and experimental data of the literature. Finally, the generalization of the method to other solute transport problems will be illustrated.

\section{Physical description and governing equations.}

In the Bridgman method, growth is achieved by pulling the sample through a gradient furnace. The furnace orientation and pulling direction may be horizontal or vertical depending on specific technological constraints. At the level of the growth interface, the thermal gradient is roughly uniform, and parallel to the sample axis (adiabatic zone). Heat is brought to the sample in the hot zone (inward thermal flux), and extracted in the cold zone (outward thermal flux). The sample is contained in a crucible which may have different shapes and aspect ratios (generally the shape is rectangular in the horizontal method and cylindrical in the vertical method). The solute which is rejected builds up in front of the growth interface, thus forming a solute boundary layer, and is transported into the bulk liquid by diffusion and/or convection. The physical system is schematically shown in figure 1 .

In order to write down the governing equations, the classical following assumptions are made :

The liquid is Newtonian, Boussinesq approximation is valid, linear phenomenological relations apply with no cross coupling, and transport properties do not depend on temperature and concentration. In addition, the solute content is supposed to be sufficiently low so that density differences are only due to temperature differences. Then, the field equations for mass, momentum, heat and solute in the liquid can be written as follows :

$$
\begin{gathered}
\nabla \cdot \mathbf{V}=0 \\
-\beta_{\mathrm{T}}\left(T-T_{0}\right) \mathbf{g}-\rho_{0}^{-1} \nabla p_{\mathrm{d}}+ \\
+v \nabla^{2} \mathbf{V}-(\mathbf{V} \cdot \nabla) \mathbf{V}=0 \\
\alpha \nabla^{2} T-\mathbf{V} \cdot \nabla T=0 \\
D \nabla^{2} C-\mathbf{V} \cdot \nabla C=0
\end{gathered}
$$

where $: \mathbf{V}$ is the fluid velocity vector, $p_{\mathrm{d}}$ the dynamic pressure, $T$ the temperature, $C$ the solute concentration, $\rho_{0}$ the fluid density at $T_{0}, \beta_{\mathrm{T}}$ the thermal expansion coefficient, $g$ the gravity vector, $v$ the kinematic viscosity, $\alpha$ the thermal diffusivity, and $D$ the solute diffusion coefficient.

In order to simplify the notations, we will consider a two-dimensional domain and a planar growth

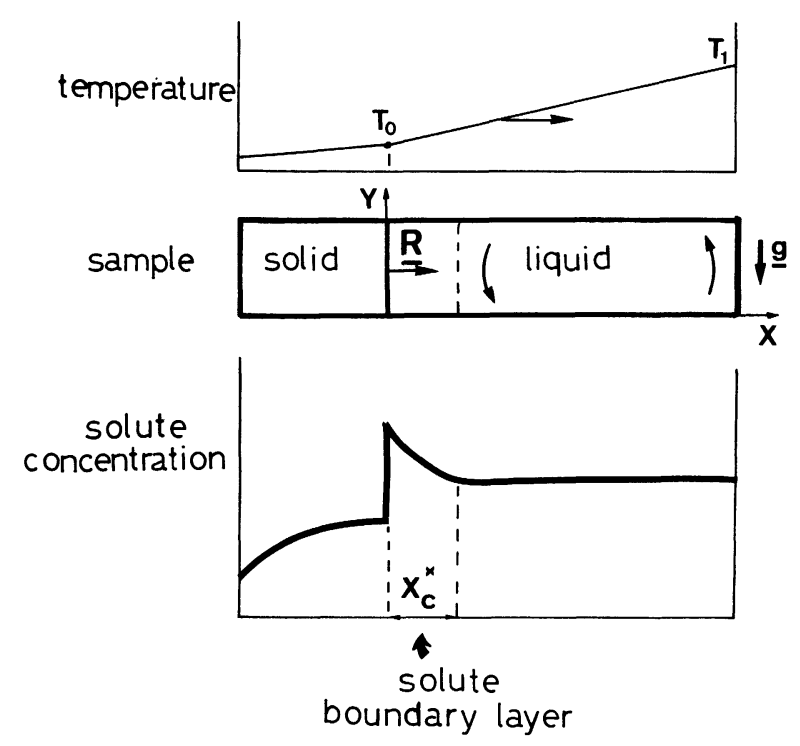

Fig. 1. - Diagram of the growth of a doped crystal from the melt by the Bridgman method (horizontal case). 
interface. $X$ and $Y$ will be the coordinates respectively perpendicular and parallel to the interface (Fig. 1) and, $U$ and $V$ the corresponding components of the velocity vector $\mathbf{V}$. This is not a fundamental restriction for the scaling analysis to be performed below : Napolitano [9] showed that the mathematical formulation could be generalized to a three dimensional domain and a non-planar interface.

With these notations, the balance equations at the growth interface are written :

$$
\begin{gathered}
U(0, Y)=-R \\
V(0, Y)=0 \\
T(0, Y)=T_{M} \\
D\left(\frac{\partial C}{\partial X}\right)_{X=0}+R\left[C\left(0^{+}, Y\right)-C\left(0^{-}, Y\right)\right]=0
\end{gathered}
$$

where $R$ is the growth rate, $T_{M}$ the melting point, $C\left(0^{+}, Y\right)$ and $C\left(0^{-}, Y\right)$ are the solute concentrations at the interface in the liquid and solid respectively.

Thermodynamic equilibrium is supposed at the solid-liquid interface :

$$
\frac{C\left(0^{-}, Y\right)}{C\left(0^{+}, Y\right)}=k
$$

where $k$ is the equilibrium partition ratio.

The other boundaries of the melt are supposed to be solid and impermeable and various thermal conditions can be applied along them.

Let $H$ be a characteristic dimension, $T_{0}$ and $T_{1}$ two characteristic temperatures. Introducing the following arbitrary set of non-dimensional quantities :

$$
\begin{aligned}
& x=\frac{X}{H}, \quad y=\frac{Y}{H}, \quad \mathrm{v}=\mathrm{V} \frac{H}{v}, \\
& \theta=\frac{T-T_{0}}{T_{1}-T_{0}}, \quad \pi=\frac{H^{2}}{\rho_{0} v^{2}} p_{\mathrm{d}}
\end{aligned}
$$

and arbitrary non-dimensional concentrations $c$, equations (1a) and (1b) are transformed into the following non-dimensional form :

$$
\begin{gathered}
\nabla \cdot \mathbf{v}=0 \\
-G r \theta \frac{\mathbf{g}}{|g|}-\nabla \pi+\nabla^{2} \mathbf{v}-(\mathbf{v} \cdot \nabla) \mathbf{v}=0 \\
\nabla^{2} \theta-\operatorname{Pr} \mathbf{v} \cdot \nabla \theta=0 \\
\nabla^{2} c-S c \mathbf{v} \cdot \nabla c=0
\end{gathered}
$$

and :

$$
\begin{aligned}
& u(0, y)=-\operatorname{Re} \\
& v(0, y)=0 \\
& \theta(0, y)=\theta_{M} \\
&\left(\frac{\partial c}{\partial x}\right)_{x=0}+\operatorname{Pe}\left[c\left(0^{+}, y\right)-c\left(0^{-}, y\right)\right]=0
\end{aligned}
$$

with :

$$
\begin{gathered}
G r=\frac{\beta_{\mathrm{T}}|g|\left(T_{1}-T_{0}\right) H^{3}}{v^{2}}, \quad \text { (Grashof number) } \\
\operatorname{Pr}=\frac{v}{\alpha} \text { (Prandtl number) } \\
S c=\frac{v}{D} \text { (Schmidt number) } \\
\operatorname{Re}=R \frac{H}{v} \text { (Reynolds number) } \\
P e=\operatorname{ReS}(\text { (Peclet number). }
\end{gathered}
$$

Thus, the general problem depends on five dimensionless parameters ( $\mathrm{Gr}, \mathrm{Pr}, \mathrm{Sc}, \mathrm{Pe}$ and $k$ ), and the type of geometry of the crucible and thermal conditions along the crucible wall.

However, since we are presently interested in the crystal growth of metals or semi-conductors from their impure melt, we will restrict to the limiting case :

$$
\operatorname{Pr} \ll 1, \quad S c \gg 1 \text {. }
$$

For such conditions, there is a wide data range where convective solute transport occurs while heat transfer remains mainly conductive. Advantage of the resulting simplification will be taken below.

\section{Thermal buoyancy driven flow regimes.}

With the assumptions made in the previous section (sufficiently low concentration level), the flow field is not coupled to the concentration field and thus can be studied separately. We first consider the fluid motion as a function of $G r$ when the interface is at rest $(R e=0)$. Many studies have been devoted to this problem : a detailed description of the flow field corresponding to simple geometries and boundary conditions has thus been obtained either analytically with the help of simplifying physical assumptions [10-12], or numerically for a limited range of the parameter $G r$ [13]. In the present paper, our concern is in defining simple laws giving the main characteristics of the flow field, especially near the growth interface, for various geometries and boundary conditions and a wide $G r$ range. Specifically this means : define the range of validity of the Stokes flow approximation, analyse the occurrence of boundary layer flow and the corresponding scaling law for the flow velocity, choose the characteristic dimension $H$ and temperature difference $\Delta T$ defining the controlling parameter $G r$ as a function of boundary conditions, estimate the constant factors entering the scaling laws.

With this aim in view, we apply the principles of scaling analysis to the presently considered internal flow configuration, and compare the a priori estimate with the available analytical and numerical data respectively for the case of Stokes flow and boundary layer flow. We thus show that a simple and consistent set of practical laws can be defined for a wide Gr range. 
Finally we consider the fluid motion when the growth interface is moving.

3. 1 StoKes FLow. - Classically, for $G r \rightarrow 0$, the non-linear convective term can be neglected in the Navier-Stokes (N.S.) equations. If $v^{*}$ is a characteristic flow velocity, inertia forces of order $v^{* 2}$ are smaller than viscous forces of order $v^{*}$, provided that :

$$
v^{*} \ll 1 \text {. }
$$

Then viscous and buoyancy forces are of the same order :

$$
v^{*} \sim G r .
$$

In other words, replacing the unknown $v$ by $v^{\prime}=v / G r$ simply eliminates the parameter $G r$ from the problem.

For given geometry and boundary conditions, the solutions corresponding to different $\mathrm{Gr}$ values are simply similar to one another. Choosing as a reference the fluid velocity in a characteristic point (for instance, the maximum velocity $v_{\mathrm{m}}$ ) one gets :

$$
v_{\mathrm{m}}=\frac{1}{K} G r \text {. }
$$

The value of constant $K$ depends on the geometry, boundary conditions, choosen reference temperature difference $\Delta T$ and dimension $H$. For the simple case of the two-dimensional flow in a square enclosure :

$$
K=144 \sqrt{3}
$$

according to the solution given by Batchelor [10]. In the case of complex geometries and boundary conditions, a physically meaningful choice of $\Delta T$ and $H$ must be made if one wants to get good estimates of the fluid velocity by comparison with simple cases. This point is discussed below.

Let us first consider a horizontal rectangular enclosure with a very large aspect ratio $A=L / H$ ( $L$ and $H$ respectively length and height) and a horizontal applied thermal gradient, which corresponds to a horizontal Bridgman crystal growth. At low Gr, a unique convective cell is formed, the flow is onedimensional in the central part and turns round in end regions of extent $H$ [13]. Thus the characteristic dimension to be considered is the small dimension $H$ and the temperature difference is the one at this length scale. With these references and according to the analytical solution given by Birikh [11] :

$$
K=72 \sqrt{3} \text {. }
$$

If the same horizontal $\Delta T$ is appliéd across a vertical slot of width $H$, the calculated flow field has an identical form.

In the case of vertical Bridgman crystal growth, a stabilizing vertical thermal gradient is applied. However, as discussed in detail in [21], horizontal gradients cannot be avoided because of technological constraints. If both types of gradients are present, the analytical model of Birikh [12] for the flow in a vertical layer of width $H$ between two differentially heated plates shows that the stabilizing effect of the vertical gradient $G$ is only effective when :

$$
R a=\frac{\beta_{\mathrm{T}} g G H^{4}}{v \alpha} \gg 100 \text { (Rayleigh number) } .
$$

Thus, considering the low values of $\operatorname{Pr}$ for metals, we can neglect this stabilizing effect in a first approximation. The problem then reduces to the horizontal case.

However, the geometry and boundary conditions corresponding to vertical Bridgman crystal growth are more complex than those of the Birikh model. The sample is generally cylindrical and the thermal field roughly has a cylindrical symmetry. As a consequence, the vertical and horizontal components of the temperature gradient are not uniform along the sample axis. A first attempt to treat a realistic situation was made by Chang and Brown [17]. As already discussed in [21], the calculations show that, for sufficiently low driving forces, relation (5b) applies with $G r$ defined from the radius of the sample and the maximum radial temperature difference and $K \simeq 30$. In this model, however, temperatures were directly imposed at the melt-crucible boundary, which leads to overestimate the radial temperature differences in the hot zone of the sample.

In fact, as schematically shown in figure 2 , the inward thermal flux is reduced in the hot zone of the sample due to the limited exchange between furnace and crucible and the thermal resistance of the crucible itself. In addition, aspect ratios may be large $(A \geqslant 10)$. Thus, it may be necessary to distinguish different regions in the melt where the driving forces for the flow are different. Particularly, as shown by more

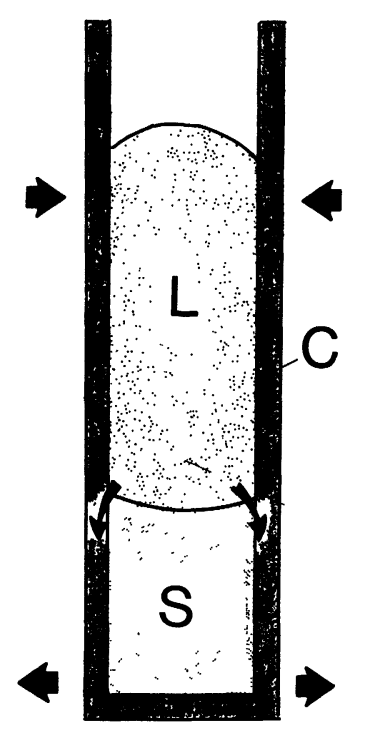

Fig. 2. - Diagram of the vertical Bridgman crystal growth : $\mathrm{S}$ : solid phase; $\mathrm{L}$ : liquid phase; $\mathrm{C}$ : crucible; arrows represent thermal fluxes. 
recent simulations [18] which consider more realistic boundary conditions, a secondary cell can be formed in the vicinity of the growth interface, either inertially driven by the bulk flow or directly associated with local gradients generated by the difference between the conductivities of the solid and the liquid phases (Fig. 2 illustrates the case where the solid conductivity is the lowest (semiconductors)).

From the previously discussed global characteristics of the flow (number of cells, flow direction and velocity) one can estimate the flow in the vicinity of the growth interface, i.e. for $x \ll 1$. Indeed, expanding both components of velocity in terms of $x$, and applying the no-slip condition at the interface and the continuity equation, one gets at first order :

$$
\begin{aligned}
& v=v_{0}(y) x \sim v^{*} x \\
& u=u_{0}(y) x^{2} \sim v^{*} x^{2}=v x .
\end{aligned}
$$

For comparison the Batchelor solution [10] gives :

$$
\begin{aligned}
& \max _{0<y<1} v(x, y)=\frac{G r}{24} x=\frac{v_{\mathrm{m}}}{0.096} x \\
& \max _{0<y<1} u(x, y)=\frac{G r}{18 \sqrt{3}} x^{2}=\frac{v_{\mathrm{m}}}{0.125} x^{2} .
\end{aligned}
$$

3.2 BOUNDARY LAYER FLOW. - Scaling analysis shows that for sufficiently large $G r$, inertia forces (of order $v^{* 2}$ ) dominate viscous forces (of order $v^{*}$ ) in the core of the liquid. Then the velocity scale $v^{*}$ is given by a balance between buoyancy and inertia :

$$
v^{*} \sim G r^{1 / 2}
$$

and pressure is of order of inertia forces :

$$
\pi^{*} \sim v^{* 2} .
$$

However, when the viscous terms are neglected, the order of the N.S. equations is reduced and the compatibility with boundary conditions is no longer ensured.

Thus, there is a region near the boundaries where the viscous forces cannot be neglected (momentum boundary layer). This introduces a new length scale $x^{*}$ in the direction normal to the boundary. As a result of the no-slip condition at the boundary and the continuity equation, the scale of the velocity component $u^{*}$ normal to the boundary is in this boundary layer region :

$$
u^{*}=v^{*} x^{*}
$$

With these new scales the non-dimensional N.S. equations become :

$$
\begin{aligned}
& -\frac{\pi^{*}}{x^{* 2}} \frac{\partial \pi^{\prime}}{\partial x^{\prime}}+\frac{v^{*}}{x^{* 2}}\left(\frac{\partial^{2} u^{\prime}}{\partial x^{\prime 2}}+x^{* 2} \frac{\partial^{2} u^{\prime}}{\partial y^{2}}\right)-v^{* 2}\left(u^{\prime} \frac{\partial u^{\prime}}{\partial x^{\prime}}+v^{\prime} \frac{\partial u^{\prime}}{\partial y}\right)=0 \\
& G r \theta-\pi^{*} \frac{\partial \pi^{\prime}}{\partial y}+\frac{v^{*}}{x^{* 2}}\left(\frac{\partial^{2} v^{\prime}}{\partial x^{\prime 2}}+x^{* 2} \frac{\partial^{2} v^{\prime}}{\partial y^{2}}\right)-v^{* 2}\left(u^{\prime} \frac{\partial v^{\prime}}{\partial x^{\prime}}+v^{\prime} \frac{\partial v^{\prime}}{\partial y}\right)=0
\end{aligned}
$$

where $x^{\prime}, u^{\prime}, v^{\prime}$ and $\pi^{\prime}$ denote the new dimensionless variables.

Only with such a non-dimensional form, the scale of the different terms within the boundary layer can be given by the corresponding non-dimensional coefficients. With this form, $x^{*}$ can be defined by writing that the dominant tangential viscous forces $\left(\frac{v^{*}}{x^{* 2}}\right)$ are of the order of inertia forces $\left(v^{* 2}\right)$ (see for instance [7]) :

$$
v^{*} x^{* 2} \sim 1
$$

Combining with (7) gives :

$$
x^{*} \sim G r^{-1 / 4} .
$$

Taking into account the no-slip condition at the interface, the tangential velocity field $v(x)$ in the vicinity of the interface can be estimated as :

$$
v(x) \sim \frac{v^{*}}{x^{*}} x=G r^{3 / 4} x
$$

and using the continuity equation, the normal velocity field $u(x)$ is given by :

$$
u(x) \sim v(x) \cdot x=G r^{3 / 4} x^{2} .
$$

Figure 3 shows the variation of the flow velocity scale $v^{*}$ as a function of $\mathrm{Gr}$ (dashed lines) together with the available numerical data on the maximum flow velocity $v_{\mathrm{m}}$ for different geometries (full lines). Both types of data are quite close to one another in the high $\mathrm{Gr}$ range which indicates that the concept of a boundary layer flow remains valid even in the present internal flow configuration. Moreover, contrary to the Stokes flow regime, the constant factor entering the scaling law for $v$ in this regime appears to be close to 1 . This can be qualitatively understood by considering that, in the boundary layer regime, there is no direct viscous interaction between the flows along the different boundaries of the enclosure, but only a weak interaction through the core.

The transition from the Stokes flow to the boundary layer flow (Navier-Stokes regime) can be defined as the $G r$ value for which the curves $v_{\mathrm{m}}=\frac{1}{K} G r$ and $v=G r^{1 / 2}$ intersect. For the horizontal bidimensional 


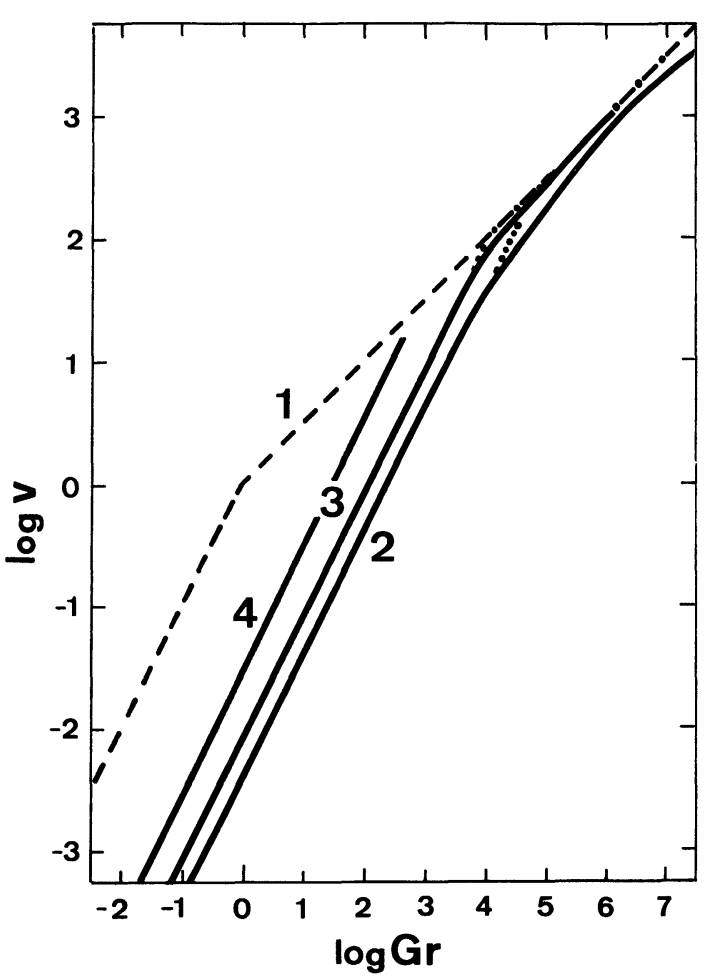

Fig. 3. - Plot of the flow velocity $v$ versus $G r: 1$ : a priori scaling laws (relations (5) and (7)), 2-3-4 : analytical and numerical data for different geometries and aspect ratios $A$ : 2 : square enclosure $(A=1)[10,16], 3:$ rectangular enclosure $(A>2)$ [11, 14], 4 : vertical cylinder $(A \cong 4)$ (with $G r$ defined from the radius and the maximum horizontal temperature difference) [17].

slot this occurs for $G r \simeq 10^{4}$. This value compares very well with the stability limit of the unidimensional flow in this geometry : $G r=7920$ [12].

3.3 Practical Characteristic QUANTITIES. - According to the discussion of the previous section, the flow can be simply described in terms of orders of magnitude with the help of two characteristic quantities, a velocity $v^{*}$ and a boundary layer extent $x^{*}$. These quantities are defined by relations (5a), (7) and (11) which can be summarized by the following constrained maximum problem as written by Napolitano [7] :

$$
\left\{\begin{array}{l}
\max \left(x^{*}\right) \quad x^{*} \leqslant 1 \\
\frac{G r}{v^{*}} x^{* 2}=1 \\
v^{*} x^{* 2} \leqslant 1
\end{array}\right.
$$

which means that $x^{*}$ is the maximum length scale for which viscous forces are of the same order as buoyancy forces, and at least of the order of inertia forces. The corresponding flow field in the vicinity of the boundaries is described by (6).

However, in order to get a quantitative law for a practical quantity such as the maximum flow velocity, a constant factor lower than one has to be introduced in the case of the Stokes flow regime (relation (5b)). This can be understood by considering that, due to the viscous interaction that occurs in this regime between the flows along the different boundaries, the length scale available for the flow is not the overall dimension of the cell but only a fraction $x_{0}$ of it. Thus, a consistent set of practical characteristic quantities can be defined by the following modified form of (14) :

$$
\left\{\begin{array}{l}
\max \left(x^{*}\right) \quad x^{*} \leqslant x_{0} \\
\frac{G r}{v^{*}} x^{* 2}=1 \\
v^{*} x^{* 2} \leqslant 1 .
\end{array}\right.
$$

The solution of system (14b) is given in table $\mathrm{I}$. The constant $x_{0}$ depends on the geometry of the problem, and can be defined by the condition that $v^{*}$ equals the maximum flow velocity in the Stokes regime, i.e. :

$$
x_{0}=K^{-1 / 2} \text {. }
$$

Taking into account relations (6a) and (13), a consistent description of the velocity components as a function of the distance from the boundary (growth interface) is given by :

$$
\begin{aligned}
v(x) & =\frac{v^{*}}{x^{*}} x & & \text { if } x<x^{*} \\
& =v^{*} & & \text { if } x \geqslant x^{*} \\
u(x) & =v(x) \cdot x & & \text { if } x<1 \\
& =v^{*} & & \text { if } x \geqslant 1 .
\end{aligned}
$$

If $x_{0}$ is determined by (15) which ensures that (5b) is satisfied, then relation (6b) is only approximately satisfied.

\subsection{FLOW WITH A MOVING GROWTH INTERFACE. -} We now consider that the growth interface is moving $(R e \neq 0)$. The no-slip condition at the interface is replaced by $u=-R e$, i.e. there is an imposed velocity relative to the growth interface.

The flow velocity relative to the growth interface

Table I. - Thermal buoyancy driven flow regimes in an enclosure as predicted from $(14 b)$ : the scaling laws of the momentum boundary layer $x^{*}$ and the maximum flow velocity $v^{*}$ are given for the different ranges of the parameter $G r$. The constant $x_{0}$ depends on the geometry of the enclosure and is given by (15).

\begin{tabular}{lccc}
\multicolumn{1}{c}{ Regime } & Domain & $x^{*}$ & $v^{*}$ \\
\hline Stokes flow & $G r<x_{0}^{-4}$ & $x_{0}$ & $x_{0}^{2} G r$ \\
Boundary layer flow & $G r>x_{0}^{-4}$ & $G r^{-1 / 4}$ & $G r^{1 / 2}$
\end{tabular}


is now given by :

$$
\begin{aligned}
& u(x)=\max \left(\operatorname{Re}, u_{1}(x)\right) \\
& v(x)=v_{1}(x)
\end{aligned}
$$

where $u_{1}(x)$ and $v_{1}(x)$ are the velocity components corresponding to the case $R e=0$, and are given by (16).

There is always a region near the interface where the imposed velocity is larger than the normal velocity induced by the flow in the bulk. This region will be termed a "stagnant " layer. In this layer, the solute is transported by pure diffusion.

The extent $x_{1}^{*}$ of the stagnant layer is solution of :

$$
u\left(x_{1}^{*}\right)=\operatorname{Re}
$$

where $u\left(x_{1}^{*}\right)$ is given by (16) where $x^{*}$ and $v^{*}$ are taken from table I.

Particularly, the whole liquid is «stagnant " if :

or,

$$
G r<x_{0}^{-4} \text { and } x_{0}^{2} G r<R e
$$

$$
G r>x_{0}^{-4} \text { and } G r^{1 / 2}<R e .
$$

This is a sufficient condition for the solute transport to be diffusive (regardless of the value of $S c$ ). This is not however a necessary condition as will be analysed in detail in the next section.

\section{The solute boundary layer : scaling.}

Taking into account the main characteristics of the buoyancy driven flows derived in the previous section, a scaling analysis of the solute transport during crystal growth from an impure melt can be performed. This analysis will be performed in two steps : first we will study the stationary solute profile which would be reached in the melt if the sample were of infinite length; then we will consider the real situation of a sample of finite length.

4.1 The Stationary CaSe (THE INFINITE SAMPLE). In the ideal situation where a sample of infinite length is solidified, the boundary conditions for solute transport do not depend on time. Then, as far as time dependent convective regimes are not considered, the solute profile in the liquid is stationary and the solute concentration in the crystal is constant along the growth axis.

4.1.1 The reference diffusive case. - When there is no convection $(\mathrm{Gr}=0)$, the flow velocity relative to the growth interface is simply :

$$
u=-R e \text {. }
$$

Introducing an unspecified characteristic length scale $\delta_{\mathrm{c}}$ along the growth direction, the dimensionless solute balance equation ( $3 \mathrm{~d})$ becomes :

$$
\frac{\partial^{2} c}{\partial x^{\prime 2}}+x_{c}^{* 2} \frac{\partial^{2} c}{\partial y^{2}}+P e x_{c}^{*} \frac{\partial c}{\partial x^{\prime}}=0
$$

with $x_{\mathrm{c}}^{*}=\frac{\delta_{\mathrm{c}}}{H}$.

Classically, if the concentration only depends on $x$, there is a solute boundary layer in front of the growth interface, the extent of which is given by :

$$
P e \cdot x_{c}^{*}=1
$$

or $\delta_{\mathrm{c}}=D / R$.

The concentration in the crystal is uniform and equal to the concentration in the melt at infinite distance from the interface $c_{\infty}$. And the concentration in the melt at the interface is : $c(0)=\frac{c_{\infty}}{k}$. Variations of $c$ along the $y$ direction (hereafter referred to as radial segregation) can only be caused by a curvature of the solidification front. According to [28], these obey the following approximate relation :

$$
\Delta c \cong(1-k) \frac{R}{D} \Delta X
$$

where $\Delta X$ is the maximum variation of $x$-coordinates along the interface.

4.1.2 Convective case : the solute boundary layer. When convection is present in the melt, diffusive transport always dominates in a region near the interface where the no-slip condition applies. In the same way as for momentum transport, the solute boundary layer $x_{\mathrm{c}}^{*}$ can be defined as the scale at which the diffusive and convective contributions are of the same order of magnitude. In order to scale the convective contribution, the normal and tangential components of the flow velocity have to be evaluated within the solute boundary layer, i.e. they are given by (17) where $x$ is taken equal to $x_{\mathrm{c}}^{*}$. The resulting nondimensional form of the solute balance equation is :

$$
\begin{aligned}
\frac{\partial^{2} c}{\partial x^{\prime 2}}+x_{\mathrm{c}}^{* 2} \frac{\partial^{2} c}{\partial y^{2}}-S c u\left(x_{\mathrm{c}}^{*}\right) x_{\mathrm{c}}^{*} u \frac{\partial c}{\partial x^{\prime}}- & \\
- & -S c v\left(x_{\mathrm{c}}^{*}\right) x_{\mathrm{c}}^{* 2} v^{\prime} \frac{\partial c}{\partial y}=0 .
\end{aligned}
$$

Since :

$$
\operatorname{Scu}\left(x_{\mathrm{c}}^{*}\right) x_{\mathrm{c}}^{*} \geqslant \operatorname{Scv}\left(x_{\mathrm{c}}^{*}\right) x_{\mathrm{c}}^{* 2}
$$

The condition defining $x_{\mathrm{c}}^{*}$ simply reads :

$$
\operatorname{Scu}\left(x_{\mathrm{c}}^{*}\right) x_{\mathrm{c}}^{*}=1 .
$$

In order to solve (24), $u\left(x_{\mathrm{c}}^{*}\right)$ is evaluated by using (17), (16) and table I. Then, according to the data range, different regimes appear, i.e. different scaling laws are obtained for $x_{\mathrm{c}}^{*}$ or equivalently for the segregation parameter $\Delta$ defined by :

$$
\Delta=P e \cdot x_{\mathrm{c}}^{*} .
$$


Table II. - Solute transport regimes during Bridgman crystal growth as predicted from (24), (17), (16) and table I : The scaling laws of the solute boundary layer $x_{\mathrm{c}}^{*}$ are given for the different ranges of the parameters, $\mathrm{Pe}, \mathrm{Gr}$ and $\mathrm{Sc}$, the corresponding domains being drawn in figure 4.

\begin{tabular}{lll} 
Regime & \multicolumn{1}{c}{ Domain } & \multicolumn{1}{c}{$x_{\mathrm{c}}^{*}$} \\
\hline III & $\begin{array}{l}P e<A^{-1} \\
x_{0}^{2} G r S c<A^{-1}\end{array}$ & $A$ \\
II & $\begin{array}{l}\text { complement to III and I } \\
\text { I.a }\end{array} A^{-1}<x_{0}^{2} G r S c<1$ & $\begin{array}{l}P^{-1} \\
\left(x_{0}^{2} G r S c\right)^{-1}\end{array}$ \\
I.b & $\begin{array}{l}P e<x_{0}^{2} G r S c \\
1<x_{0}^{2} G r S c<x_{0}^{-2}\end{array}$ & $\left(x_{0}^{2} G r S c\right)^{-1 / 2}$ \\
I.c & $\begin{array}{l}P e<\left(x_{0}^{2} G r S c\right)^{1 / 2} \\
x_{0}^{-4}<G r S c<x_{0}^{-4} S c\end{array}$ & $\left(x_{0} G r S c\right)^{-1 / 3}$ \\
I.d & $\begin{array}{l}P e<\left(x_{0} G r S c\right)^{1 / 3} \\
x_{0}^{-4}<G r\end{array}$ & $G r^{-1 / 4} S c^{-1 / 3}$ \\
& $P e<G r^{1 / 4} S c^{1 / 3}$ &
\end{tabular}

The results are shown in table II where the different ranges are defined in terms of the parameters $P e, G r$ and $S c$, and the associated scaling laws of $x_{\mathrm{c}}^{*}$ presented. The corresponding domains are drawn in figure 4 for the particular case : $S c=10, A=10, K=100$. Regime III, which appears in the case of a sample of finite length to be treated in section 4.2, will not be considered here.

Then, two main regimes have to be distinguished :

(II) When the thickness of the "stagnant " layer is larger than the diffusion length, we recover the diffusive regime previously described $(\Delta=1)$.

(I) In the opposite case, i.e. when the normal flow velocity in the diffusion layer is larger than the growth rate, there is a convective transport regime. The solute boundary is smaller than the diffusion length $(\Delta<1)$ and decreases when convection increases.

It can be noticed that domain (II) is larger than the domain defined by (19), i.e. there is a domain where the maximum flow velocity is larger than the growth rate and, however, solute transport is diffusive.

Inside domain (I), several sub-regimes can be distinguished. Three of them correspond to situations where there is no momentum boundary layer $(\mathrm{Gr}<$ $x_{0}^{-4}$ ). Then there is only a solute boundary layer, the extent of which decreases as convective mixing increases :

$$
\begin{aligned}
& 1<x_{\mathrm{c}}^{*} \\
& x_{0}<x_{\mathrm{c}}^{*}<1 \\
& x_{\mathrm{c}}^{*}<x_{0} .
\end{aligned}
$$

In all three cases, the scaling laws only depend on the product $G r S c$.

Finally, for $G r>x_{0}^{-4}$, there is both a solute and a momentum boundary layer (domain (I.d)) and the problem formally (although weakly) depends on $\mathrm{Gr}$ and $S c$ independently.

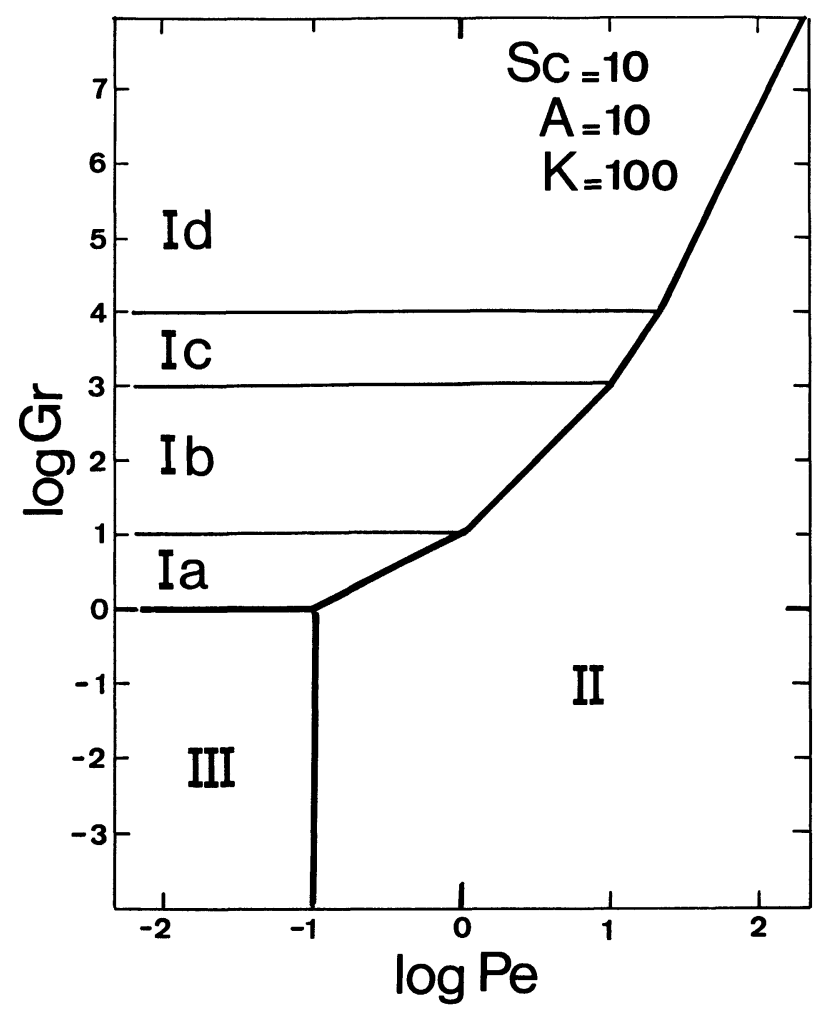

Fig. 4. - Domains corresponding to the different solute transport regimes : II diffusive boundary layer regime. I convective boundary layer regime

$$
\begin{array}{lrl}
\text { I. }: ~: 1<x_{\mathrm{c}}^{*} & x^{*}=x_{0} \\
\text { I. } \mathrm{b}: x_{0}< & x_{\mathrm{c}}^{*}<1 & x^{*}=x_{0} \\
\text { I.c : } \quad x_{\mathrm{c}}^{*}<x_{0} & x^{*}=x_{0} \\
\text { I.d : } \quad x_{\mathrm{c}}^{*}<x_{0} & x^{*}<x_{0}
\end{array}
$$

III fast diffusion regime.

4.1.3 Convective case : the concentration scale. In the analysis above, the solute boundary layer has been defined without considering the solute balance at the interface (3h). Thus an arbitrary concentration scale could be used and the definition of the solute boundary layer did not depend on the partition ratio $k$. Equation ( $3 \mathrm{~h})$ in fact determines the scale $c(0)$ of the concentration at the interface. Indeed, considering that :

$$
\left(\frac{\partial c}{\partial x}\right)_{0} \sim \frac{c(0)-c_{\infty}}{x_{c}^{*}}
$$

equation $(3 \mathrm{~h})$ gives :

$$
\frac{c(0)}{c_{\infty}} \sim \frac{1}{1-(1-k) \Delta} .
$$

Thus the effective partition ratio $k_{\text {eff }}$ reads :

$$
k_{\text {eff }}=\frac{c_{\mathrm{s}}}{c_{\infty}}=\frac{k}{1-(1-k) \Delta} .
$$


The radial segregation $\Delta c$ induced by the convective motion is at most of order $c(0)-c_{\infty}$. Thus :

$$
\frac{\Delta c}{c(0)} \sim(1-k) \Delta
$$

This expression is only valid if $x_{\mathrm{c}}^{*}<1$.

When $x_{\mathrm{c}}^{*}>1, \Delta c$ is only of order $c(0)-c(1)$, and :

$$
\frac{\Delta c}{c(0)} \sim(1-k) P e .
$$

In the diffusive regime, the radial segregation induced by the residual convective motion will be proportional to the ratio of the flow velocity to the growth rate. Thus, radial segregation is maximum at the transition between the convective and the diffusive transport regime.

4.2 LONGITUDINAL SEGREGATION (THE FINITE SAMPLE). - Since in practice the solidified sample has not an infinite length, there are two reasons for a departure from the stationary conditions described in the previous section :

(i) solidification starts from an initial liquid of uniform concentration,

(ii) the melt has a finite length.

Thus, if the predicted solute boundary layer extent $x_{\mathrm{c}}^{*}$ is larger than the total melt length $A$, the melt is homogenized at any time by diffusion. This can be readily verified by evaluating the scale of the concentration at the interface from the solute balance at the interface (Eqs. (3)), taking into account that :

$$
\left(\frac{\partial c}{\partial x}\right)_{0} \sim \frac{c(0)-c(A)}{A}
$$

This is the fast diffusion regime (domain III of table II and Fig. 4). In the course of solidification, the solute concentration in the melt changes according to the classical Scheil equation. And a typical normal segregation profile results in the crystal along the growth axis.

In the convective regime, an identical normal segregation profile will be obtained although for a quite different physical reason (the liquid phase now being homogenized by convection). However, it must be noticed that this time dependence of the concentration at the scale of the whole sample does not imply that the conditions of solute transport are not locally stationary. This local stationarity will be ensured as long as the time derivative term remains negligible in the local solute balance.

At last, in the diffusive solute boundary layer regime (domain II), a sub-regime must be distinguished. Since solidification starts from an initial liquid of uniform concentration, the solute has to build up in front of the interface at the beginning of solidification until it reaches the stationary concentration $c_{\infty} / k$. Thus, a longitudinal segregation will appear in the crystal due to this initial transient for $A^{-1} \leqslant P e \leqslant A^{-1} k^{-1}$ (domain II').

The different types of segregation obtained as a function of the data range are summarized in figure 5 . Quantitative profiles can be obtained by using a unique boundary layer model as described in [3].

\section{The solute boundary layer : comparison with nume- rical and experimental data.}

Most data refer to the growth of the semiconductor $\mathrm{Ge}$ doped with $\mathrm{Ga}\left(10^{17}\right.$ to $\left.10^{19} \mathrm{at} / \mathrm{cm}^{3}\right)$. The corresponding physical properties are recalled in table III. Numerical simulations of the horizontal Bridgman crystal growth have been performed by Polezhayev [14] The geometry is bidimensional and rectangular, and typical values of the dimensionless parameters are $: S c=10, k=0.087, A=4, P e=5, G r=10^{2}$,

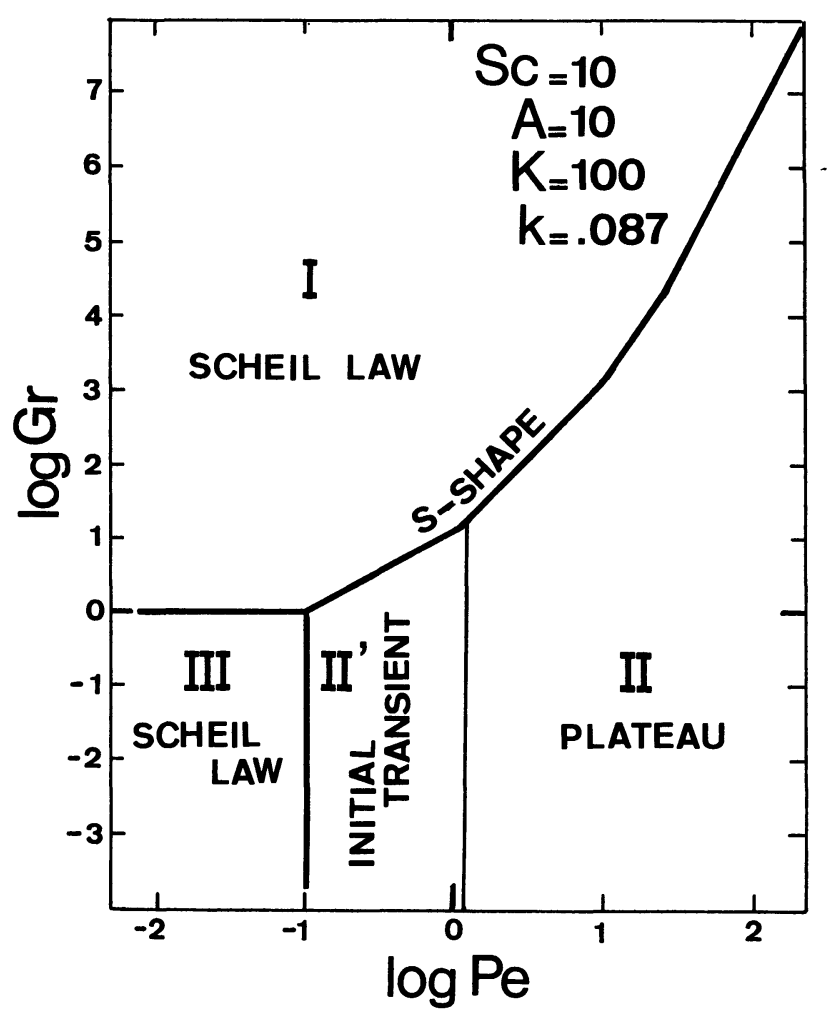

Fig. 5. - Types of longitudinal segregation profiles as a function of the data range.

Table III. - Physical properties of $\mathrm{Ge}$ doped with $\mathrm{Ga}$.

Thermal diffusivity : $\quad \alpha=0.2 \mathrm{~cm}^{2} \mathrm{~s}^{-1}$

Thermal expansion

coefficient :

$\beta_{\mathrm{T}}=1.1 \times 10^{-4} \mathrm{~K}^{-1}$

Kinematic viscosity : $v=1.3 \times 10^{-3} \mathrm{~cm}^{2} \mathrm{~s}^{-1}$ [29]

Chemical diffusivity : $\quad D=1.9 \times 10^{-4} \mathrm{~cm}^{2} \mathrm{~s}^{-1}[30]$

Equilibrium partition

ratio : $\quad k=0.087$ 
$10^{3}, 10^{4}$. The calculations are fully time-dependent and take into account the initial transient and the change of the melt length in the course of solidification, which is representative of a real crystal growth situation. Thus a complete map of the dopant concentration in the crystal is obtained, including both the radial and longitudinal segregation. For the simulations made in $[14,15], \Delta$ has been calculated by fitting the curve of the mean concentration in vertical slices with the one dimensional boundary layer model. The values of $\Delta$ thus obtained are compared in figure 6 with the scaling laws derived in section 4 . This shows that our analysis correctly predicts the transition from diffusive to convective transport with increasing $G r$. Unfortunately, calculations are not available for sufficiently high $G r$ values to check the validity of the scaling law in the convective regime. Figure 6 only indicates that a constant factor of about 2 or 3 should be incorporated to our scaling law in this range. Finally, the simulations in [14] show that the radial segregation goes through a maximum for $G r \sim 10^{2}$, which is near to the transition between the diffusive and convective transport regimes (break of the $\Delta$ curve in Fig. 6).

Chang and Brown [17] simulated the transport of a dopant in the vertical Bridgman configuration already described in section 3.1. The problem was made stationary with the help of a fictitious boundary condition at the top of the melt. Thus, the radial segregation corresponding to stationary growth conditions was obtained. Some estimates of $k_{\text {eff }}$ and $\Delta$ were also made from the analysis of the mean solute profile in the liquid along the growth axis. A large number of runs have been made which cover a significant range of values of $\mathrm{GrSc}$ and $\mathrm{Pe}$. The variation of the calculated $\Delta c / c$ with $G r$ is compared with our predictions in figure 7 for

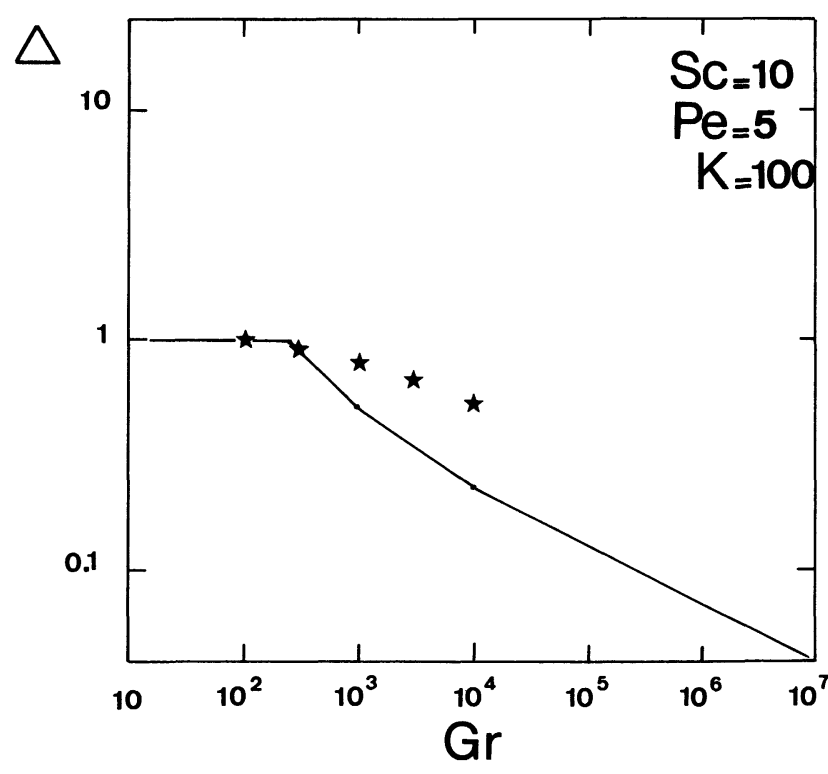

Fig. 6. - Comparison of the a priori scaling laws of $\Delta$ and the numerical simulations $[14,15](\star)$.

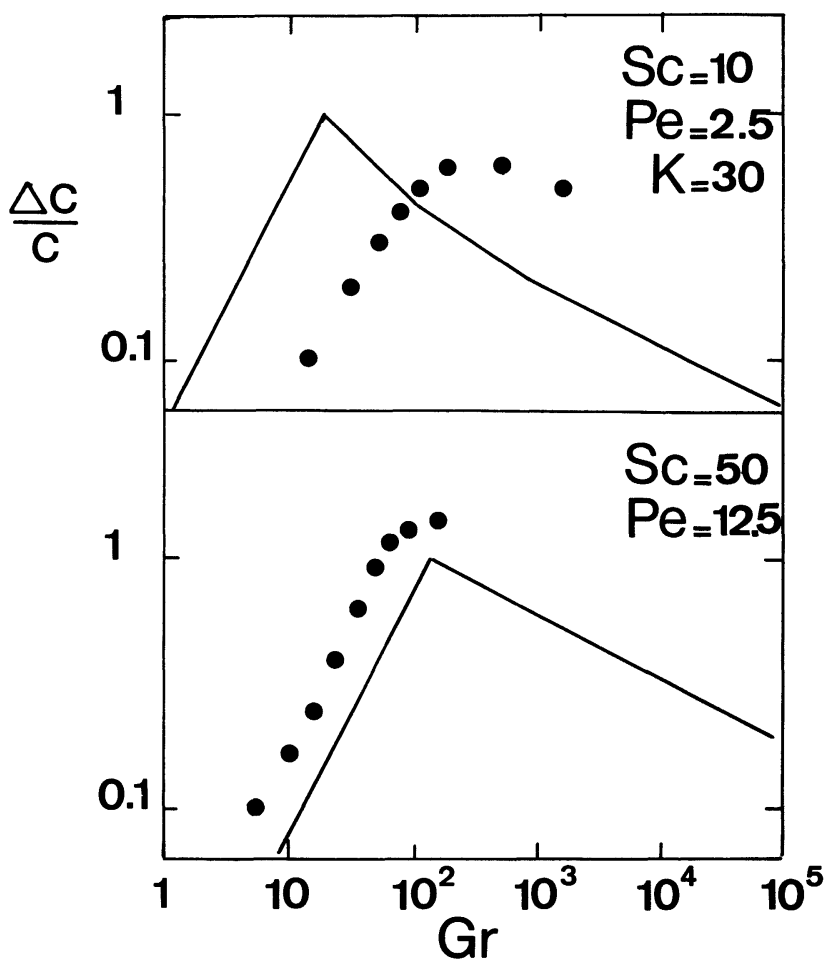

Fig. 7. - Comparison of the a priori scaling laws of $\Delta c / c$ and the numerical simulations [17] (๑).

two different $P e$ and $S c$ values. In addition, curves of iso- $\Delta c / c$ are drawn in figure 8 . This illustrates that $\Delta c / c$ goes through a maximum in the range of transition from the convective to the diffusive regime. The damping of the effect for low $G r S c$ and $P e$ values (i.e. for large solute boundary layers) is also clear. But figure 8 would indicate that our analysis underestimates this effect. However, no quantitative conclusion should be drawn due to the particular top boundary condition considered in the simulations. At last, the simulations predicted a direction of the radial segregation leading to a $\mathrm{Ga}$ enrichment at the periphery of the sample. This is the opposite of the experimental findings. The reason for this lies in the oversimplification of the thermal boundary conditions as discussed in section 3.1. Only the more recent simulations considering more realistic boundary conditions [18] predict the correct direction for radial segregation.

Growth experiments on $\mathrm{Ga}$ doped $\mathrm{Ge}$ have been performed by different authors in various thermal conditions on the ground in the vertical configuration or in microgravity conditions [19-21, 32]. Precise measurements of the $\mathrm{Ga}$ concentration profiles in the crystals have been performed by the spreading resistance method and $\Delta$ has been calculated by fitting the experimental segregation profile with a boundary layer model. The growth conditions and experimental results are given in table IV, and compared with our a priori estimates. For comparison we made two different estimates $\Delta_{1}$ and $\Delta_{2}$ for $\Delta$ from our analysis, by considering horizontal temperature differences 


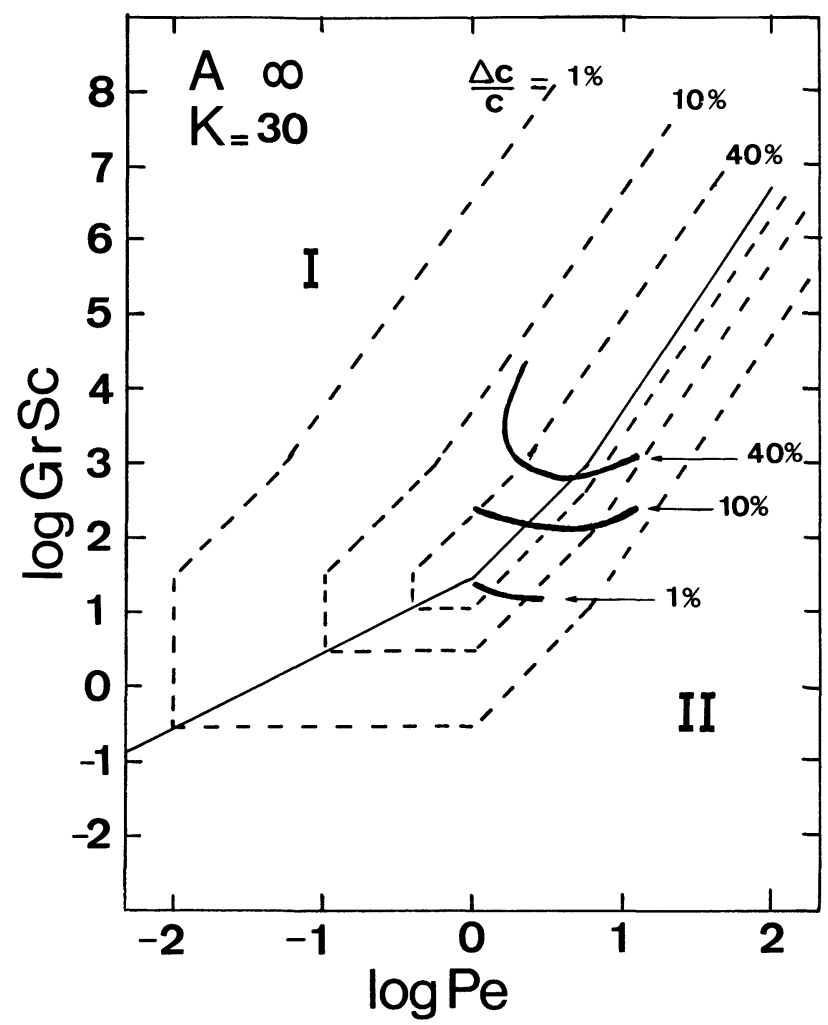

Fig. 8. - Comparison of the predicted iso- $\Delta c / c$ curves (dashed lines) with those calculated in [17] (full lines).

given by :

and

$$
\Delta T_{1}=G . r
$$

$$
\Delta T_{2}=G . \Delta X
$$

where $G$ is the longitudinal thermal gradient, $r$ is the radius of the ampoule and $\Delta X$ the observed vertical deflection of the growth interface, which can be considered as an isotherm.
Table IV shows that for Witt's experiment [19] the measured $\Delta$ is consistent with the a priori estimate $\Delta_{1}$ if it is considered that, as suggested by figure $6, \Delta_{1}$ has to be multiplied by a constant factor of about 2 . The amplitude of radial segregation is also consistent with $\Delta_{1}$. However, even in this experiment, the observed radial segregation corresponds to a $\mathrm{Ga}$ enrichment near the axis of the sample. This shows that a secondary convective cell, mainly forced by the motion in the bulk liquid, should be present in the vicinity of the crystal-melt interface.

In the other experiments, it appears that the radial temperature difference to be considered should be lower than $\Delta T_{1}$ (experiments of Wang [20] and Favier [32]) and even $\Delta T_{2}$ (experiments of Rouzaud [21]). This decrease of the effective radial temperature difference can be ascribed to the reduction of the radiative exchanges between the furnace and sample (use of a non-transparent crucible), and the extension of the adiabatic zone of the furnace by a special design of the heating element.

Thus, transport conditions can easily be predicted by our analysis in the case of a horizontal or pseudohorizontal crystal growth configuration. In the case of the vertical thermally stabilizing configuration, more experimental data would be needed in order to get precise correlations, since small differences in the applied boundary conditions can drastically change the values of the radial temperature differences and their location. However, generally speaking, it can be concluded that, in order to achieve a diffusive solute transport in the melt, very low residual horizontal temperature differences would be required, which cannot be achieved in practice.

Such diffusive transport conditions can only be achieved in a microgravity environment. The maximum allowed stationary $g$-level in any direction non parallel to the growth axis can then be determined with our analysis. Thus, both the microgravity experiments by Witt [19] and Favier [22, 32] fall within our

Table IV. - Experimental data on Bridgman crystal growth of $\mathrm{Ga}$ doped $\mathrm{Ge}:$ Comparison of the experimental values of $\Delta$ derived from the segregation curves $\left(\Delta_{\text {exp }}\right)$, with the a priori estimates derived from table II using (28)

\begin{tabular}{|c|c|c|c|c|c|c|c|c|c|}
\hline Reference & $g / g_{0}$ & $\begin{array}{c}r \\
(\mathrm{~cm})\end{array}$ & $\begin{array}{c}G \\
\mathrm{~K} \mathrm{~cm}^{-1}\end{array}$ & $\begin{array}{c}R \\
\mathrm{~cm} \mathrm{~s}^{-1}\end{array}$ & $\begin{array}{l}\Delta X \\
(\mathrm{~cm})\end{array}$ & $\Delta_{\exp }$ & $\Delta c / c$ & $\Delta_{1}$ & $\Delta_{2}$ \\
\hline \multirow[t]{2}{*}{ [19] } & 1 & 0.5 & 50 & $8 \times 10^{-4}$ & 0.03 & 0.142 & 0.25 & 0.05 & 0.1 \\
\hline & $10^{-5}$ & 0.5 & 50 & $8 \times 10^{-4}$ & 0.03 & $\sim 1$ & $\sim 1$ & 1 & 1 \\
\hline \multirow[t]{3}{*}{ [20] } & 1 & 0.68 & 60 & $2 \times 10^{-4}$ & 0.06 & 0 & 0.06 & 0.012 & 0.021 \\
\hline & 1 & 0.68 & 60 & $4 \times 10^{-4}$ & 0.06 & 0.13 & 0.15 & 0.024 & 0.043 \\
\hline & 1 & 0.68 & 60 & $8 \times 10^{-4}$ & 0.06 & 0.27 & 0.35 & 0.048 & 0.085 \\
\hline \multirow[t]{2}{*}{ [21] } & 1 & 0.4 & 55 & $8 \times 10^{-5}$ & 0.02 & 0.1 & 0 & 0.006 & 0.013 \\
\hline & 1 & 0.4 & 55 & $8 \times 10^{-4}$ & 0.02 & 1 & 0.3 & 0.06 & 0.13 \\
\hline [32] & $\begin{array}{l}1 \\
10^{-4}\end{array}$ & $\begin{array}{l}0.25 \\
0.25\end{array}$ & $\begin{array}{l}50 \\
50\end{array}$ & $\begin{array}{l}3.5 \times 10^{-3} \\
3.5 \times 10^{-3}\end{array}$ & 0.055 & 0.778 & 0.5 & 0.22 & 0.32 \\
\hline
\end{tabular}
$\left(\Delta_{1}\right.$ and $\left.\Delta_{2}\right)$. 
domain II, consistently with the observed longitudinal profile (initial transient and plateau). However, in all experiments, a radial segregation has been observed. This should have been caused mainly by the diffusion in front of a curved solidification interface [28]. However, a contribution of the residual buoyancy cannot be excluded, particularly for Witt's experiment which falls not far from the transition between domains I and II.

\section{Generalization of the method.}

The method presented above can be extended to analyse the contribution of convection to solute transport in other processes. First we will consider the case where the buoyancy driving force is replaced by forced convection. This corresponds to the classical Czochralski growth technique for which the solute boundary layer concept had been first developed. Then as a first approximation the buoyancy term can be neglected in the N.S. equations, and the boundary condition at the interface is modified to :

$$
V=\omega H
$$

where $\omega$ is the rotation rate of the crystal and $H$ its radius. This gives in dimensionless form :

$$
v=R e_{\omega}
$$

with

$$
R e_{\omega}=\frac{\omega H^{2}}{v}
$$

The criterion(14) determining $v^{*}$ and $x^{*}$ now becomes :

$$
\left\{\begin{array}{l}
\max \left(x^{*}\right) \quad x^{*} \leqslant x_{0} \\
v^{*} x^{* 2} \leqslant 1 \\
\frac{R e_{\omega}}{v^{*}}=1
\end{array}\right.
$$

And the solute boundary layer $x_{c}^{*}$ is solution of :

$$
\left\{\begin{array}{l}
\max \left(x_{\mathrm{c}}^{*}\right) \quad x_{\mathrm{c}}^{*}<\frac{L}{H} \\
\operatorname{Scu}\left(x_{\mathrm{c}}^{*}\right) \cdot x_{\mathrm{c}}^{*}=1
\end{array}\right.
$$

where $L$ is the melt depth (which for the sake of simplicity is presently considered equal to the crystal radius : $L \cong H)$, and $u\left(x_{\mathrm{c}}^{*}\right)$ is given by (17).

The solutions of (17), (29) and (30) have been reported in table V. Analytical data are available in the literature in the case of the momentum and solute boundary layer regimes $[1,23,24]$. These data are in accordance with the scaling laws of table $\mathrm{V}$. The corresponding constant factors, which have also been reported in the table, appear not to be very different from one. Thus our analysis qualitatively generalizes previous results.

The same method can be used to analyse situations where several convective driving forces are involved, for instance buoyancy and forced convection in Czochralski growth. Then, the last relation in (29) is replaced by :

$$
\max \left(\frac{R e_{\omega}}{v^{*}}, \frac{G r}{v^{*}} x^{* 2}\right)=1 .
$$

Table V. - Momentum and solute transport regimes during Czochralski crystal growth as predicted from (17), (29) and (30): the scaling laws of the flow velocity $v^{*}$ and the momentum and solute boundary layers $x^{*}$ and $x_{\mathrm{c}}^{*}$, are given for the different ranges of the parameters $R e, R e_{\omega}$ and $S c$. Results of analytical and numerical studies are also given when available.

Flow regimes Domain

$$
x^{*}
$$

1
1
$R e_{\omega}^{-1 / 2}$
$0.35 \underset{e_{\omega}^{-1 / 2}}{\operatorname{Re}^{-1}}$

$$
\begin{aligned}
\operatorname{Re} & <\operatorname{Re} e_{\omega}<1 \\
\operatorname{Re} e_{\omega} & <\operatorname{Re}<1 \\
1 & <\operatorname{Re} e_{\omega} \\
\operatorname{Re} & <\operatorname{Re} e_{\omega}^{1 / 2} \\
1 & <\operatorname{Re} \\
\operatorname{Re} e_{\omega}^{1 / 2} & <\operatorname{Re}
\end{aligned}
$$

Solute transport regimes
Domain

$$
P e<1
$$$$
R e_{\omega} S c<1
$$

II

I.c complement to III and I

$$
\begin{gathered}
1<R e_{\omega} S c<S c \\
P e<\left(R e_{\omega} S c\right)^{1 / 3} \\
1<R e_{\omega} \\
P e<R e_{\omega}^{1 / 2} S c^{1 / 3}
\end{gathered}
$$

$v^{*}$

$\begin{array}{rl} & R e_{\omega} \\ & R e \\ & R e_{\omega} \\ 0.18 & R e_{\omega}[23] \\ & R e^{2}\end{array}$


Thus, in the case of momentum boundary layer regimes, the classical condition for an equivalence between buoyancy and forced convection $[25,26]$ is recovered :

$$
R e_{\omega}^{2}=G r .
$$

Using (30), a consistent description of the solute boundary layer extent $\delta$ can be obtained for all possible values of $R e$ and $G r$. A refined analysis should however take into account that the length scales for forced convection and buoyancy may not be the same if the radius of the crystal and crucible are very different.

A further analysis should include the possible effect of Marangoni convection at the melt surface. Such a discussion however is out of the scope of the present paper.

Finally, the proposed method can be used to analyse the effect of convection on other solute transport phenomena, for instance thermomigration [27].

\section{Conclusions.}

The present paper showed that scaling analysis could be successfully applied to the study of momentum and solute transport in Bridgman crystal growth from the melt.

Concerning the flow, simple and reasonably accurate laws have been established, which predict the transition from Stokes flow to boundary layer flow and give the main characteristics of the flow field, especially in the vicinity of the growth interface.

The method was then applied to the solute transport by using the previously determined flow field characteristics. The validity of the concept of a solute boun- dary layer $x_{\mathrm{c}}^{*}$ was confirmed. The different solute transport regimes were defined as a function of $\mathrm{Gr}, \mathrm{Pe}$ and $S c$. The corresponding scaling laws for $x_{c}^{*}$ were obtained, and the types of segregation induced in the crystal predicted.

As has been illustrated, these results of our analysis can be used for instance to :

- rationalize scarce experimental and numerical data corresponding to various systems and boundary conditions,

- predict the parameter ranges for which simplifying physical assumptions can be made and particular solutions obtained,

- select precise data and boundary conditions for which numerical computations can be most useful,

- provide data to enter analytical boundary layer models of segregation phenomena,

- define the thermal boundary conditions to be applied in order to achieve better controlled experiments,

- specify the maximum allowed stationary $g$-level in a spatial experiment where pure diffusion control is desired.

Finally, the present paper shows that scaling analysis can be usefully associated to experimental, analytical and numerical work in the study of convective phenomena in crystal growth.

\section{Acknowledgments.}

Special thanks are given to Dr. A. Rouzaud for fruitful discussions in the course of the work. The present work was supported by CEA-IRF and CNES under joint contract GRAMME.
[1] Polezhaev, V. I., in Crystals : growth, properties and applications 10 (Springer-Verlag, Berlin) 1984, p. 87-150.

[2] Burton, J. A., Prim, R. C. and Slichter, W. P., J. Chem. Phys. 21 (1953) 1987.

[3] FAVIER, J. J., Acta Met. 29 (1981) 197 and 205.

[4] FAVIER, J. J. and Wilson, L. O., J. Crystal Growth 58 (1982) 103.

[5] Schlichting, H., Boundary Layer Theory, 7th Ed. (McGraw-Hill, New York) 1979.

[6] Ostrach, S., Ann. Rev. Fluid Mech. 14 (1982) 313.

[7] Napolitano, L. G., Acta Astronautica 9 (1982) 199.

[8] Camel, D. and Favier, J. J., J. Crystal Growth 67 (1984) 42 and 57.

[9] Napolitano, L. G., L'Aerotecnica Missili E Spazio (dec. 1977) 183.

[10] Batchelor, G. K., Quart. Appl. Math. 12 (1954) 209.

[11] BirIKH, R. V., J. Appl. Mech. Tech. Phys. 3 (1966) 43.

[12] Cited by GershunI, Convective stability of incompressible fluids, Israel Program for Scientific Transla- tions, Jerusalem 1976, p. 290.

[13] HART, J. E., Int. J. Heat Mass Transfer 26 (1983) 1069.

[14] Polezhaev, V. I. et al., J. Crystal Growth 52 (1981) 465 and 471.

[15] Polezhaev, V. I., personal communication.

[16] Stewart, M. J., Weinberg, F., J. Crystal Growth 12 (1972) 217.

[17] Chang, C. J. and Brown, R. A., J. Crystal Growth 63 (1983) 343.

[18] Adornato, P. and Brown, R. A., to be published.

[19] Witt, A. F., Gatos, H. C., Lichtensteiger, M. and Herman, C. J., J. Electrochem. Soc. 125 (1978) 1832.

[20] WANG, C. A., Ph. D. Thesis, MIT Boston, Feb. 1984.

[21] Kouzaud, A., Camel, D. and Favier, J. J., J. Crystal Growth 73 (1985) 149.

[22] Favier, J. J. et al., Acta Astronautica 9 (1982) 255.

[23] Wilson, L. O. and SCHRYER, N. L., J. Fluid Mech. 85 (1978) 479.

[24] Wilson, L. O., J. Crystal Growth 44 (1978) 247. 
[25] Jones, A. D. W., Progress in Crystal Growth and Characterization $9(1,2)$ (Ed. B. R. Pamplin, Pergamon, Oxford) 1984, p. 139.

[26] Mihelcic, M., Wingerath, K. and Pirron, C., J. Crystal Growth 69 (1984) 473.

[27] Malmejac, Y. and Praizey, J. P., Proceedings of the 5th European Symposium on Material Sciences under Microgravity, Schloss Elmau, 5-7 nov. 1984 (ESA SP-222) 147 to 152.
[28] Coriell, S. R., Boisvert, R. F., ReHM, R. G. and SEKERKA, R. F., J. Crystal Growth 54 (1981) 167. [29] Smithells, C. J., Metals Reference Book, 5th Ed. (Butterworths, London) 1976.

[30] Bourret, E. D., Favier, J. J. and Bourrel, O., J. Electrochem. Soc. 128 (1981) 2437.

[31] Hansen, M., Constitution of Binary Alloys (McGrawHill, New York) 1958

[32] FAVIER, J. J. and DE GOER, J., C.E.A. Internal Report, Grenoble 1985. 